\title{
THE TOPOLOGY OF THE SPACE OF DENJOY INTEGRABLE FUNCTIONS
}

\author{
Chew Tuan Seng and Lee Peng Yee
}

In this paper, the topology of the Denjoy space and characterisation of precompact sets in the Denjoy space are given.

\section{INTRODUCTION}

Let $D$ be the Denjoy space, that is, the space of all Denjoy integrable (in the restricted sense) functions defined on a closed compact interval $[a, b]$ of the real line $[8$, p.30; 10, p.241]. It is well-known that the space $D$ can be normed by

$$
\|\mid f\|=\sup \left\{\left|\int_{a}^{x} f(t) d t\right| ; a \leqslant x \leqslant b\right\} .
$$

Unfortunately, it is not complete under this norm and is of the first category [11]. Recently several convergence theorems, namely, the controlled convergence theorem, the generalised mean convergence theorem and the generalised dominated convergence theorem, have been proved for the space $D$, see $[5,6,7,8]$. These convergence theorems are in some sense better than the classical dominated convergence theorem. For example, using the generalised mean convergence theorem, we may define the Denjoy integral by means of a sequence of step functions $[6 ; 8, p .58]$, and, as we shall see in this paper, a topology by means of generalised mean convergence.

Let $\Delta=\left\{X_{i}\right\}$ be a sequence of closed subsets of $[a, b]$ with $X_{i} \subset X_{i+1}$ and $a, b \in X_{i}$, for all $i$, and $[a, b]=\bigcup_{i} x_{i}$. Let $J$ be the family of all such $\triangle$. For each $\triangle \in J$, let $D(\triangle)$ be the space of all Denjoy integrable functions defined on $[a, b]$ such that its primitive function is of bounded variation on each $X_{i}$. Clearly, $D=U_{\Delta} D(\Delta)$. In this paper, we shall show that each $D(\Delta)$ is a complete, separable, metrisable locally convex space with a topology generated by generalised mean convergence. Some applications on operators and characterisation of precompact sets in $D(\Delta)$ are also given.

Received 15 January 1990

Copyright Clearance Centre, Inc. Serial-fee code: 0004-9729/90 \$A2.00+0.00. 


\section{Preliminaries}

In this section, we state some known results which will be used later.

Definition 2.1: $[6 ; 7 ; 8, p .55]$. A sequence of functions $\left\{f_{n}\right\}$ in the space $D$ is said to be generalised mean convergent if

(i) there exists a sequence of closed sets $X_{i}$ such that $[a, b]=\bigcup_{i} X_{i}$ and for every $i$ and $\varepsilon>0$, there exists a natural number $N$ such that for any partial division $P=\{[u, v]\}$ of $[a, b]$ with all $u, v \in X_{i}$, we have

$$
(P) \Sigma \omega\left(F_{n}-F_{m},[u, v]\right)<\varepsilon
$$

whenever $n, m \geqslant N$, where $\Sigma$ is over the division $P, F_{n}$ is the primitive of $f_{n}$ and $\omega$ is the oscillation of $F_{n}-F_{m}$ over $[u, v]$.

(ii) the primitives $F_{n}$ converge uniformly to $F$ on $[a, b]$.

REMARK 2.2: In the Definition 2.1, we may assume that $\Delta=\left\{X_{i}\right\} \in J$, that is, $a, b \in X_{i}$ and $X_{i} \subset X_{i+1}$, for all $i$, and use the following condition (ii)' instead of (i) and (ii),

(ii)' the condition (ii) holds with $u, v \in X_{i}$ replaced by $u$ or $v \in X_{i}$ and the inequality replaced by

$$
(P) \Sigma\left|F_{n}(v)-F_{m}(v)-F_{n}(u)+F_{m}(u)\right|<\varepsilon .
$$

It can be done since, in view of (ii), we may assume $a, b \in X_{i}$, for all $i$. Then (i) holds with $u, v \in X_{i}$ replaced by $u$ or $v \in X_{i}$ for each $i$, see [8, p.54]. Furthermore, the oscillation in the definition can be replaced by the difference, since $F_{n}$ are continuous. Therefore (i) holds with $X_{i}$ replaced by $\bigcup_{n=1}^{i} X_{n}$. Consequently, we may assume that $X_{i} \subset X_{i+1}$, for all $i$.

In the Definition 2.1, we do not assume that $\left\{f_{n}\right\}$ is pointwise convergent; for this condition see Remark 2.4.

Theorem 2.3. [7, Theorem $4 ; 8, p .55]$. If $\left\{f_{n}\right\}$ in the space $D$ is generalised mean convergent, then there exist $f \in D$ and a pointwise convergent subsequence of $\left\{f_{n}\right\}$ with the limit $f$. Furthermore,

$$
\lim _{n \rightarrow \infty} \int_{a}^{b} f_{n}(x) d x=\int_{a}^{b} f(x) d x
$$

REMARK 2.4: The function $F$ given in Definition 2.1 condition (ii), in fact, is the primitive function of $f$, since $F(x)=\lim _{n \rightarrow \infty} \int_{a}^{x} f_{n}(t) d t=\int_{a}^{x} f(t) d t$. Therefore, 
it is convenient to say that $\left\{f_{n}\right\}$ is generalised mean convergent to $f$, when $\left\{f_{n}\right\}$ is generalised mean convergent; here it is understood that the primitive function of $f$ is $F$ given in condition (ii) of Definition 2.1. Theorem 2.3 is a generalisation of the mean convergence theorem for Lebesgue integrals. As in the case of Lebesgue integrals, the sequence $\left\{f_{n}\right\}$ given in Theorem 2.3 does not necessarily converge to $f$ pointwise.

Next, we shall consider "control-convergent" which is closely related to "generalised mean convergent".

Definition 2.5: $\left[5 ; 8\right.$, p.36]. A sequence of functions $\left\{f_{n}\right\}$ in the space $D$ is said to be control-convergent to $f$ if

(i) $f_{n}(x) \rightarrow f(x)$ almost everywhere in $[a, b]$ as $n \rightarrow \infty$;

(ii) the primitives $F_{n}$ of $f_{n}$ are $A C G^{*}$ uniformly in $n$ (for the definition of $A C G^{*}$, see $[5 ; 8$, p.29]);

(iii) the primitives $F_{n}$ are equicontinuous on $[a, b]$ (for this condition, see $[7$, p.137; 8, Corollary 7.9, p.41]).

REMARK 2.6: As in Definition 2.1, the conditions (ii) and (iii) can be replaced by the following condition:

(ii)' for every $i$ and $\varepsilon>0$, there exists $\delta>0$ such that

$$
(P) \Sigma\left|F_{n}(u, v)\right|<\varepsilon \text { for all } u
$$

whenever $(P) \Sigma|v-u|<\delta$, where $P=\{[u, v]\}$ is a partial division of $[a, b]$ with $u$ or $v \in X_{i}, F_{n}$ is the primitive of $f_{n}, F_{n}(u, v)=F_{n}(v)-$ $F_{n}(u)$ and $\Delta=\left\{X_{i}\right\} \in J$.

For condition (ii)', see $\left[8\right.$, Lemma 6.4, p.27]. We say that $\left\{F_{n}\right\}$ is $A C^{*}\left(X_{i}\right)$ uniformly for each $i$ if $\left\{F_{n}\right\}$ satisfies (ii)'.

THEOREM 2.7. $\left[8\right.$, p.56, p.63]. If $\left\{f_{n}\right\}$ is control-convergent to $f$ then $\left\{f_{n}\right\}$ is generalised mean convergent to $f$. Conversely, if $\left\{f_{n}\right\}$ is generalised mean convergent to $f$, then there is a subsequence $\left\{f_{n, i}\right\}$ of $\left\{f_{n}\right\}$ which is control-convergent to $f$.

In the above theorem, all $f_{n}$ and $f$ lie in the same $D(\Delta)$. Therefore it is natural and useful to consider a topology generated by generalised mean convergent or controlconvergent sequences in the space $D(\triangle)$.

\section{ThE TOPOLOGY}

Let the space $D(\Delta)$ be fixed. Now we shall define an increasing sequence of norms on $D(\Delta)$. Let $\Delta=\left\{X_{i}\right\} \in J$ as in Section 1 , and $f \in D(\Delta)$, then its primitive $F$ is 
of bounded variation (in the restricted sense) on each $X_{i}$; thus $\|f\|_{i}^{\Delta}<\infty$, for all $i$, where

$$
\|f\|_{i}^{\Delta}=\sup _{p}(P) \Sigma|F(u, v)|,
$$

$F(u, v)=F(v)-F(u)$ and $P=\{[u, v]\}$ is any partial division of $[a, b]$ with $u$ or $v \in X_{i}$, see [8, Lemma 6.15, p.32]. Each $\|\cdot\|_{i}^{\Delta}$ is a semi-norm, and the sequence of semi-norms $\left\{\|f\|_{i}^{\Delta}\right\}$ with $f \in D(\triangle)$ is increasing, for $X_{i} \subset X_{i+1}$ for all $i$. Define

$$
\|f\|^{\Delta}=\sum_{i=1}^{\infty} \frac{1}{2^{i}} \frac{\|f\|_{i}^{\Delta}}{1+\|f\|_{i}^{\Delta}}
$$

then $\|\cdot\|^{\Delta}$ is an $F$-norm and $D(\Delta)$ is a metrisable locally convex space generated by the increasing sequence of norms $\left\{\|\cdot\|_{i}^{\Delta}\right\}$, see $[3$, pp.202-205]. More precisely,

$$
U_{i}^{\Delta}=\left\{f ;\|f\|_{i}^{\Delta}<2^{-i}\right\}, \quad \text { for } i=1,2, \ldots
$$

forms a neighbourhood base of the origin. Note that $U_{i}^{\Delta} \supset U_{i+1}^{\Delta}$, for all $i$. This type of neighbourhood has been used in [9].

THEOREM 3.1. A sequence $\left\{f_{n}\right\}$ is Cauchy (or convergent to $f$ ) in the space $D(\triangle)$ with the norm $\|\cdot\|^{\Delta}$ if and only if $\left\{f_{n}\right\}$ is Cauchy (or convergent to $f$ ) in $D(\triangle)$ with the norm $\|\cdot\|_{i}^{\Delta}$ for each $i$.

Proof: It follows from the fact that the neighbourhood $\|f\|^{\Delta}<2^{-k}, k \geqslant 1$, contains the neighourhood $\|f\|_{k+1}^{\Delta}<2^{-k-1}$; conversely, the neighbourhood $\|f\|_{m}^{\Delta}<$ $2^{-k}$ contains the neighbourhood $\|f\|^{\Delta}<2^{-m-k-1} ;$ see $[3$, p.206].

THEOREM 3.2. A sequence $\left\{f_{n}\right\}$ is $\|\cdot\|^{\Delta}$-convergent to $f$ in $D(\Delta)$ if and only if $\left\{f_{n}\right\}$ is mean-convergent to $f$ in $D(\Delta)$.

Proof: This follows from Theorem 3.1 and the definition of norm $\|\cdot\|_{i}^{\Delta}$.

THEOREM 3.3. (a) If $\left\{f_{n}\right\}$ is $\|\cdot\|^{\Delta}$-convergent to $f$ in $D(\Delta)$, then there is a subsequence $\left\{f_{n, i}\right\}$ of $\left\{f_{n}\right\}$ which is control-convergent to $f$.

(b) If $\left\{f_{n}\right\}$ is control-convergent to $f$ in $D(\Delta)$, then $\left\{f_{n}\right\}$ is $\|\cdot\|^{\Delta}$-convergent to $f$.

Proof: This follows from Theorems 2.7 and 3.2.

THEOREM 3.4. The space $D(\Delta)$ is complete under \|\|$^{\Delta}$.

Proof: Let $\left\{f_{n}\right\}$ be a Cauchy sequence in $D(\triangle)$. Then, by Theorem 3.1, the sequence $\left\{f_{n}\right\}$ is a Cauchy sequence under $\|\cdot\|_{i}^{\Delta}$, for each $i$. Thus $\left\{f_{n}\right\}$ satisfies condition (ii)' of Remark 2.2 , that is, the sequence $\left\{f_{n}\right\}$ is generalised mean convergent. 
In fact, by Remark 2.4 , the sequence $\left\{f_{n}\right\}$ is generalised mean convergent to $f$, where $F(x)=\lim _{n \rightarrow \infty} F_{n}(x)$ is the primitive function of $f$. Consequently, by Theorem 3.1 and the definition of $\|\cdot\|_{i}^{\Delta}$, we have $\left\|f_{n}-f\right\|^{\Delta} \rightarrow 0$ as $n \rightarrow \infty$.

THEOREM 3.5. The space $D(\Delta)$ is separable under $\|\cdot\|^{\Delta}$.

Proof: Let $f \in D(\Delta)$. Then there exists a generalised mean convergent sequence of step functions in $D(\triangle)$ with the limit $f$, see [7, Theorem $5 ; 8$, p.58]. Hence, by Theorem 3.2, such a sequence of step functions is $\|\cdot\|^{\Delta}$-convergent to $f$ in $D(\Delta)$. On the other hand, it is well-known that the space $L$ of all Lebesgue integrable functions on $[a, b]$ with the usual norm $\|\cdot\|_{1}$ is separable. Furthermore, it is easy to check that $L \subset D(\Delta)$ for each $\Delta$ and $\|f\|_{i}^{\Delta} \leqslant\|f\|_{1}$ for all $f \in L, \Delta \in J$ and $i$. Note that the neighbourhood $\|f\|^{\Delta}<1 / 2^{k}$, contains the neighbourhood $\|f\|_{k+1}^{\Delta}<1 / 2^{k+1}$, see $[3$, p.206]. Consequently, $D(\Delta)$ is separable. That is to say, the space of all step functions with rational values and rational endpoints is dense in $D(\triangle)$.

\section{LINEAR OPERATORS}

In this section, let $B$ denote a complete $F$-space with $F$-norm $\|\cdot\|_{B}$. We shall consider $\|\cdot\|^{\Delta}$-continuous and control-continuous operators from $D$ to $B$. The former is defined by using the $\|\cdot\|^{\Delta}$-norm topology whereas the latter is defined by using controlconvergent sequences in $D(\Delta)$. Control-continuous operators have been extensively discussed in $[1 ; 2 ; 8, p .85]$. In this section we shall prove that they are equivalent. Hence we may apply basic theorems (for example, Banach-Steinhaus theorem) of linear analysis for Banach spaces to control-continuous linear operators defined on the space $D$. As a consequence, it provides alternative proofs for some of Sargent's results on $\||\cdot|\|$-continuous linear functionals on the space $D$ without resorting to the Sargent space or what Sargent calls $\beta$-space $[11 ; 8, p .66]$.

Definition 4.1: Let $P: D \rightarrow B$. Then $P$ is said to be $\|\cdot\|^{\Delta}$-continuous if for each $\Delta \in J, P: D(\Delta) \rightarrow B$ is $\|\cdot\|^{\Delta}$-continuous, that is, $\left\|P\left(f_{n}\right)-P(f)\right\|_{B} \rightarrow 0$ as $\left\|f_{n}-f\right\|^{\Delta} \rightarrow 0$.

Definition 4.2: Let $P: D \rightarrow B$. Then $P$ is said to be control-continuous if $\left\|P\left(f_{n}\right)-P(f)\right\|_{B} \rightarrow 0$ whenever $\left\{f_{n}\right\}$ is control-convergent to $f$.

Theorem 4.3. Let $P: D \rightarrow B$. Then $P$ is $\|\cdot\|^{\Delta}$-continuous if and only if $P$ is control-continuous.

Proof: The necessity follows from Theorem 3.3(b). For sufficiency, let $P$ be control-continuous and $\left\{f_{n}\right\}$ be $\|\cdot\|^{\Delta}$-convergent to $f$. Then, by Theorem 3.3(a), for every subsequence of $\left\{f_{n}\right\}$, there exists a sub-subsequence $\left\{f_{n(i, j)}\right\}$ such that 
$\left\|P\left(f_{n(i, j)}\right)-P(f)\right\|_{B} \rightarrow 0$ as $n(i, j) \rightarrow \infty$. By reductio ad absurdum, $\left\|P\left(f_{n}\right)-P(f)\right\|_{B} \rightarrow 0$ as $n \rightarrow \infty$. Hence $P$ is $\|\cdot\|^{\Delta}$-continuous.

Theorem 4.4. (Banach-Steinhaus theorem). Let $P_{n}$ be a sequence of controlcontinuous linear operators from $D$ to $B$. If

$$
\lim _{n \rightarrow \infty} P_{n}(f)=P(f) \quad \text { for every } f \in D
$$

then $P$ is control-continuous.

Proof: This follows from Theorem 4.3 and the classical Banach-Steinhaus theorem.

REMARK 4.5: Sargent has proved Theorem 4.4 for $\||\cdot| \mid$-continuous linear operators by using the theory of the Sargent space [11]. However, it has been shown that on the space $D$, a linear functional is $\||\cdot|\|$-continuous if and only if it is control-continuous [8, p.103]. Thus Theorem 4.4 holds for $\||\cdot|\|$-continuous linear functionals. This provides an alternative proof of Sargent's result on $\||\cdot|\|$-continuous functionals without resorting to the Sargent space [11]. Recall that the norm $\||\cdot|\|$ is defined in Section 1.

\section{Precompact SETS in $D(\Delta)$}

In this section, we shall consider the space $D(\Delta)$ with $F$-norm $\|\cdot\|^{\Delta}$ topology.

A subset $A$ of $D(\Delta)$ is said to be precompact if the closure of $A$ in $D(\Delta)$ is compact. As $D(\triangle)$ is a complete metric space, the following three conditions are equivalent: (i) $A$ is precompact; (ii) $A$ is totally bounded, that is, $A$ has a finite $\varepsilon$-net for every $\varepsilon>0$; (iii) every sequence in $A$ contains a convergent subsequence with limit in $D(\Delta)$, see [3, p.26].

Now we shall characterise precompact subsets of $D(\Delta)$. Recall that $\Delta=\left\{X_{i}\right\} \in$ $J$.

Theorem 5.1. Let $A \subset D(\Delta)$. Then $A$ is precompact if and only if (i) $A$ is precompact in measure, that is, every sequence in $A$ contains a subsequence which converges in measure to a function $f$ in $D(\Delta)$; (ii) $A$ is $A C^{*}\left(X_{i}\right)$ uniformly for each $i$, where $\Delta=\left\{X_{i}\right\} \in J$, that is, for every $i$ and $\varepsilon>0$, there exists $\delta>0$ such that

$$
(P) \Sigma|F(u, v)|<\varepsilon \text { for all } f \in A
$$

whenever $(P) \Sigma|v-u|<\delta$, where $P=\{[u, v]\}$ is a partial division of $[a, b]$ with $u$ or $v \in X_{i}$ and $F$ is the primitive of $f$.

Proof: $(\Rightarrow)$ If $A$ is precompact, then every sequence in $A$ contains a $\|\cdot\|^{\Delta}$. convergent subsequence with limit $f$ in $D(\Delta)$. By Theorem $3.3(\mathrm{a})$, there exists a 
subsequence which is pointwise convergent to $f$ almost everywhere. Thus $A$ is precompact in measure. If $A$ is precompact, then $A$ is totally bounded, that is, for every $\varepsilon>0, A$ has a finite $\varepsilon$-net under the norm $\|\cdot\|^{\Delta}$. By Theorem 3.1, $A$ has a finite $\varepsilon$-net under the norm $\|\cdot\|_{i}^{\Delta}$, for each $i$. Hence for every $i$ and $\varepsilon>0$, there exists a finite sequence $g_{n}, n=1,2, \ldots, q$ such that for every $f \in A$, there exists $g_{j}$ with $\left\|f-g_{j}\right\|_{i}^{\Delta}<\varepsilon$. Hence $A$ is $A C^{*}\left(X_{i}\right)$ uniformly, in view of the following inequality,

$$
(P) \Sigma|F(u, v)| \leqslant\left\|f-g_{j}\right\|_{i}^{\Delta}+(P) \Sigma\left|G_{j}(u, v)\right|
$$

where $G_{j}$ is the primitive of $g_{j}$ and $P=\{[u, v]\}$ is a partial division of $[a, b]$ with $u$ or $v$ in $X_{i}$.

$(\Leftarrow)$ This follows from Remark 2.6 and Theorem $3.3(\mathrm{~b})$.

We remark that if $\Delta=\left\{X_{i}\right\}$ and each $X_{i}=[a, b]$, then $D(\Delta)=L$. Hence a criterion for precompactness in the space $L$ in $[4$, p.3] is a special case of Theorem 5.1. The criterion for the space $L$ is systematically used in the study of completely continuous operators in [4]. The similar criterion (Theorem 5.1) for the space $D(\Delta)$ may be used in the study of operators in the space $D$, which will be elaborated elsewhere.

\section{REFERENCES}

[1] T.S. Chew, 'Nonlinear Henstock-Kurzweil integrals and representation theorems', SEA Bull. Math. 12 (1988), 97-108.

[2] T.S. Chew, 'The superposition operators in the space of Henstock-Kurzweil integrable functions', Real Analysis Symposium, (Coleraine, 1988).

[3] G. Köthe, Topological vector spaces I (Springer-Verlag, Berlin, Heidleberg, New York, 1969).

[4] M.A. Krasnoselskii, Integral operators in spaces of summable functions (Noordhoff, 1976).

[5] P.Y. Lee and T.S. Chew, 'A better convergence theorem for Henstock integrals', Bull. London Math. Soc. 17 (1985), 557-564.

[6] P.Y. Lee and T.S. Chew, 'A Riesz-type definition of the Denjoy integral', Real Analysis Exchange 11 (1985/86), 221-227.

[7] P.Y. Lee and T.S. Chew, 'On convergence theorems for the nonabsolute integrals', Bull. Austral. Math. Soc. 34 (1986), 133-140.

[8] P.Y. Lee, Lanzhou lectures on Henstock integration (World Scientific, 1989).

[9] S. Nakanishi, 'L'intégration de Denjoy et l'intégration au moyen des espaces rangés, I-IV', Proc. Japan Acad. 32 (1956), 678-683; 33, (1957), 13-18, 265-270; 34 (1958), 96-101.

[10] S. Saks, Theory of the integral, 2nd ed (Warsaw, 1937).

[11] W.L.C. Sargent, 'On some theorems of Hahn, Banach and Steinhaus', J. London Math. Soc. 28 (1953), 438-451. 
National University of Singapore

Department of Mathematics

Kent Ridge, Singapore 0511

Republic of Singapore 